P0196

\title{
MECHANISM STUDY OF LIGHT-INDUCED VISUAL FATIGUE BASED ON PHYSIOLOGICAL PARAMETERS AND EVALUATION METHOD CONSTRUCTION

\author{
JIANQI CAl et al.
}

DOI 10.25039/x46.2019.PO196

from

CIE x046:2019

Proceedings

of the

29th CIE SESSION

Washington D.C., USA, June 14 - 22, 2019

(DOI 10.25039/x46.2019)

The paper has been presented at the 29th CIE Session, Washington D.C., USA, June 14-22, 2019. It has not been peer-reviewed by CIE.

(C) CIE 2019

All rights reserved. Unless otherwise specified, no part of this publication may be reproduced or utilized in any form or by any means, electronic or mechanical, including photocopying and microfilm, without permission in writing from CIE Central Bureau at the address below. Any mention of organizations or products does not imply endorsement by the CIE.

This paper is made available open access for individual use. However, in all other cases all rights are reserved unless explicit permission is sought from and given by the CIE.

CIE Central Bureau

Babenbergerstrasse 9

A-1010 Vienna

Austria

Tel.: +4317143187

e-mail: ciecb@cie.co.at

www.cie.co.at 


\title{
MECHANISM STUDY OF LIGHT-INDUCED VISUAL FATIGUE BASED ON PHYSIOLOGICAL PARAMETERS AND EVALUATION METHOD CONSTRUCTION
}

\author{
Cai, J-Q ${ }^{1, *}$, Hao, W-T2 ${ }^{2}$ Guo, $Y^{1}$, Wen, R-R ${ }^{2}$, Zeng, S-S ${ }^{1}$ \\ ${ }^{1}$ China National Institute of Standardization, Beijing, CHINA, ${ }^{2}$ Beijing Yangming Zhidao \\ Photoelectric Science and Technology Co., Ltd, Beijing, CHINA \\ caijq@cnis.gov.cn
}

DOI 10.25039/x46.2019.PO196

\begin{abstract}
Light-induced visual fatigue is an important issue in the field of lighting and displaying. In this study, we figure out that light-induced visual fatigue is not related to ocular structure variation but related to micro variation of physiological function, which is caused by lens assemblies' variations in the front of human eye and reflected by parameters such as high order aberrations, etc. For the objective evaluation of light-induced visual fatigue, we select useful physiological parameters, design measurement process and construct calculation formula. By practical test, it is confirmed that the novel method is accurate and reliable for the objective and quantitative evaluation of light-induced visual fatigue. This study is based on human factor experiment results with large sample capacity. A total of 1080 measurements are performed on 540 participants in experiments. It is important for the field of lighting and displaying, and promising for further insights into myopia mechanism.
\end{abstract}

Keywords: light quality, light-induced visual fatigue, ocular physiological parameters, human factor experiment, large sample capacity, subjective perception

\section{Introduction}

With sustainable growth of international myopia rate, an increasing number of researches concentrate on the field of visual fatigue, which induces myopia. Light features and ocular physiological parameters can be related by the influence of light on human eye. To improve light quality, it is important to reasonably assess light influence on visual fatigue of human eye.

Human eye is a special and complex kind of optical system, which is able to obtain clear image by self-adjusting. In poor light quality, visual fatigue is likely to be accumulated during the adjusting process especially. Optical imaging system of human eye consists of cornea, aqueous humour, crystalline lens, vitreous body, ciliary muscle and retina. Incident array enters the pupil and transports through mediums and finally reaches the retina. In various light environments, ciliary muscle is likely to make different reactions, and the crystalline lens deform with various shapes. As a result, accommodation of ciliary muscle causes focal distance change, which induces blurred vision. Perception of eye soreness/dryness is also likely to originate from energy assumption of ciliary muscle. Consequently, blurred vision and eye soreness/dryness are two main reflections of visual fatigue, and both of them originate from accommodation by ciliary muscle during visual task process (Weeber et al., 2016).

Accommodation process tends to be various in light environments with different light parameters (illuminance, correlated colour temperature, flicker, and spectral distribution). Fatigue perception is likely to be accumulated in different degrees during this process. To evaluate light-induced visual fatigue in objective method, correlation is needed between ocular physiological parameters and fatigue degree. In this paper, we aim to construct the equation for light-induced visual fatigue evaluation based on ocular physiological parameters. We combine computational simulation and large-sample human-factor experiment for the research. This is the first time that light-induced visual fatigue is evaluated based on ocular physiological parameters in objective method. 


\section{Materials and method}

In this study, we were sought to construct visual fatigue evaluation method based on ocular physiological parameters. Visual fatigue perception may be caused by either ocular structure or micro-variation of related organizations. We performed human factor experiments on ocular physiological parameters of 400 participants during their daily jobs from 9:00 a.m. to 11:00 a.m. Prior to and following the job, ocular measurements were performed on KR (keratometric radius), ACD (anterior chamber depth), AL (axial length) (Abulafia et al., 2015) and cornea thickness (CT). After the experiment, they were instructed to score for their visual fatigue perceptions with the questionnaire in Table 1. No variation is found for these 4 parameters (Figure 1a 1d). It is indicated that visual fatigue is not caused by ocular structure variation.

Table 1 - Questionnaire and basic information of participants

\begin{tabular}{cc}
\hline Questionnaire & Basic Information \\
\hline $0 \sim 1$ No fatigue & Participants number: 400 \\
1 2 Slight fatigue & Age distribution: $25 \sim 40$ \\
2 3 Obvious fatigue within tolerance & Average age: 29 \\
3 4 Obvious fatigue with complications & Male/Female: $1: 1$ \\
4 5 Severe fatigue & Diopter range: $0 \sim-5.00 \mathrm{D}$ \\
& Average diopter: $-1.10 \mathrm{D}$ \\
\hline
\end{tabular}
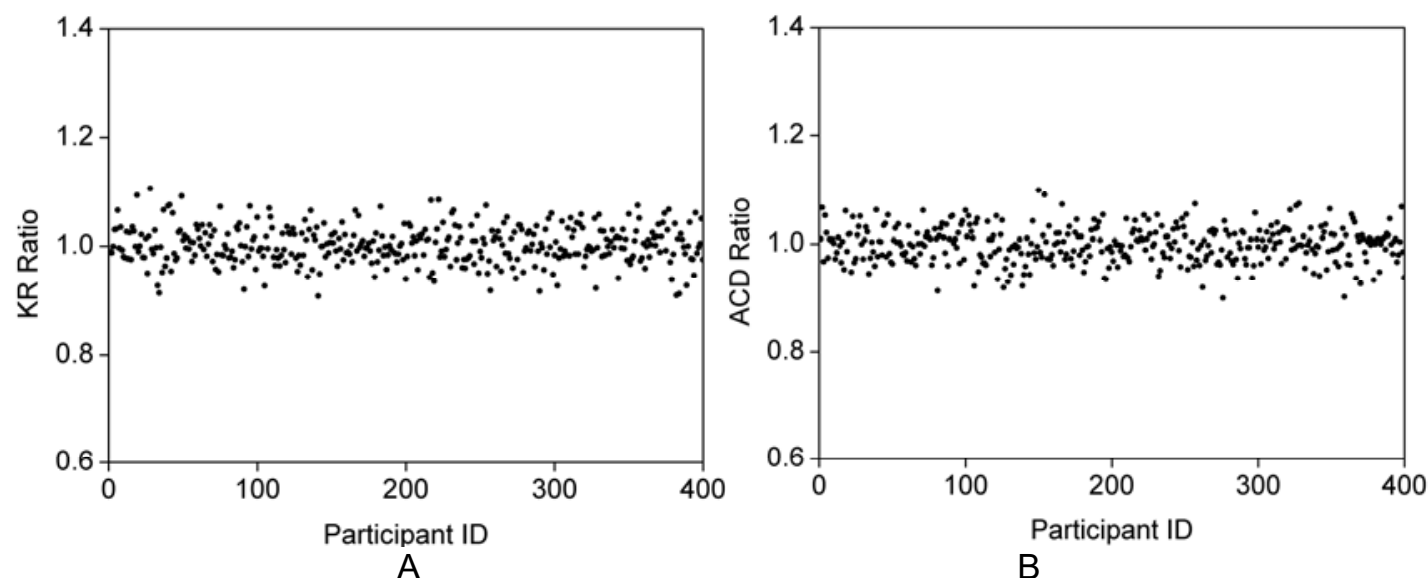

B
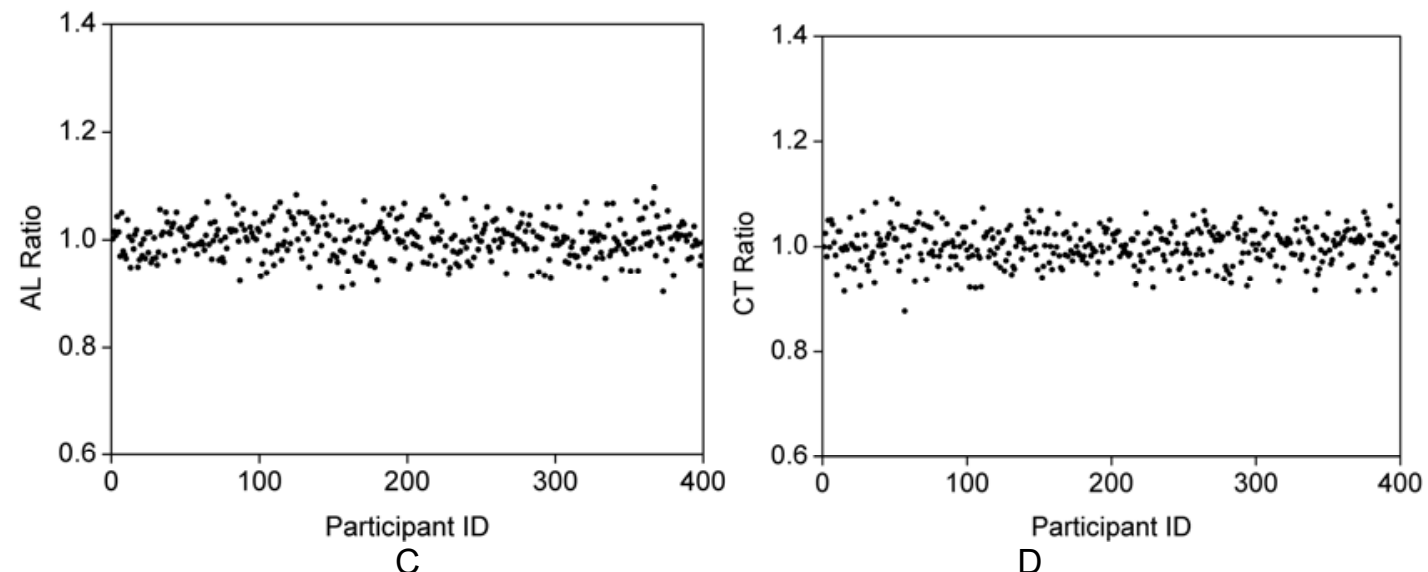

Figure 1 - Measurements of (A) KR, (B) ACD, (C) AL and (D) CT. Horizontal axes present different participants as each integer represents a participant. Vertical axes present the ratio of the final value to the initial value.

Human eye is a special optical system with an adjustable lens and a constant-focal-distance CCD camera. The adjustable optical lens consists of the cornea, ciliary muscle, crystalline lens and iris, and the retina acts as the CCD camera with constant focal distance. Fatigue is the effect of accumulation, relating to the initial and final parameters during the process. We were sought to construct the formula of visual fatigue and ocular physiological microvariations. 


\subsection{Physiological parameters}

Visual fatigue presents symptoms such as ophthalmic acid, lacrimation, blurred imaging and resolution decreasing. These symptoms are related to IOP (Intraocular pressure), and BUT (breakup time of tear film) (Norback et al., 2017), HOAs (high order aberrations) (Charman et al., 1991), MTF (modulation transfer function) (Nano et al., 2017; Charman et al., 2017, Rubert et al., 2015), AL (axial length) and ACC (accommodation).

For parameter selection, we performed stability experiment on 20 participants. All participants are free from oculopathies such as presbyopia and glaucoma, without oculopathies such as cataract, heterotropia or amblyopia. Prior to measurement, they closed eyes for $20 \mathrm{~min}$ to get a thorough rest. Following that, ocular measurements were performed. Measurements were performed three times in three different days on these 20 individuals. BUT is a good indicator, but the measurement tends to destroy the current ocular status. IOP data are quite fluctuating, only AL, ACC, HOAs and MTF are reliable (Figure 2a 2e). HOA is described by Zernike polynomial. Only the 4th and the 12th term are independent from measurement angle.
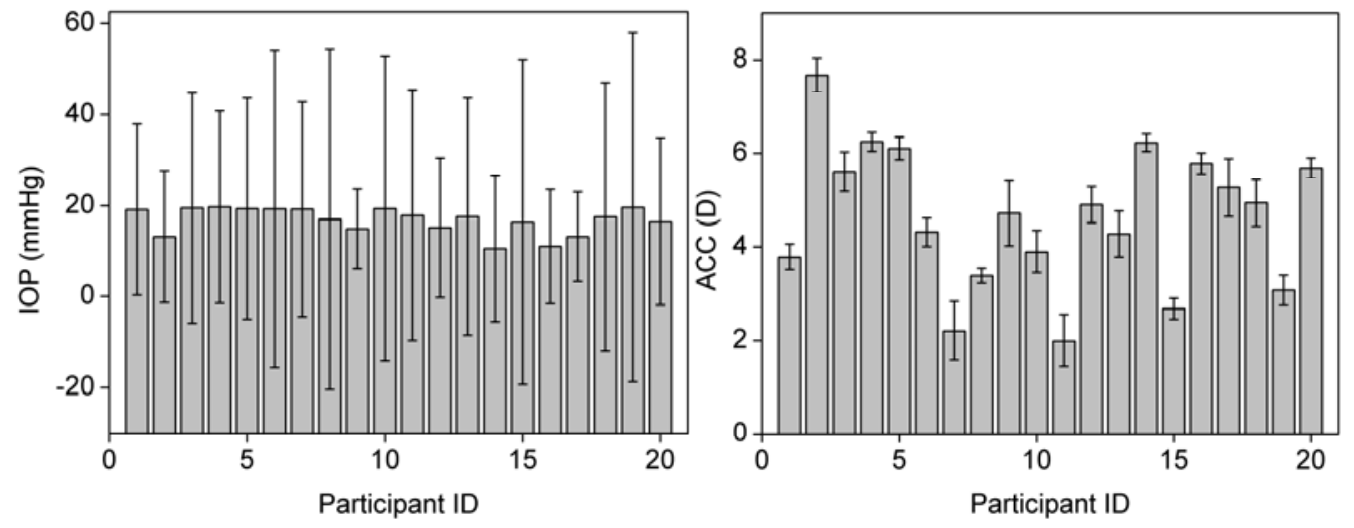

A

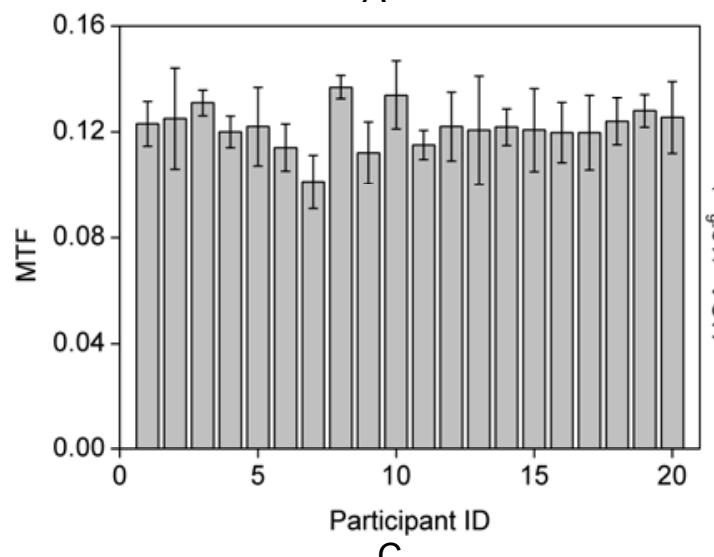

B
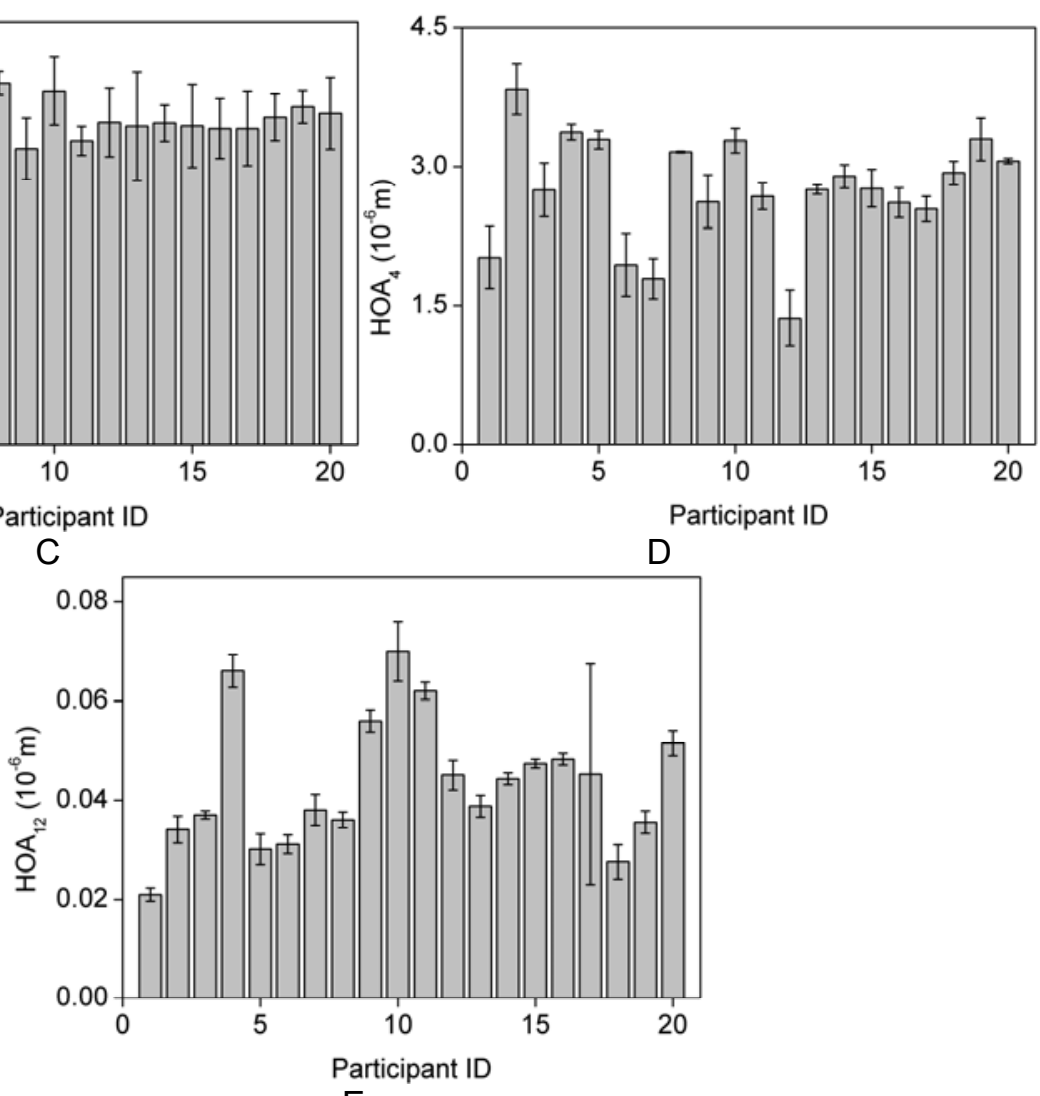

$\mathrm{E}$

Figure 2 - Stability analyses on (A) IOP, (B) ACC, (C) MTF, (D) HOA4, and (E) HOA12. The bar represents the average value after $20 \mathrm{~min}$ rest in three days. Error bar represents the standard deviation of the three days measurements. 
Then we measured light-induced variation. Participants were asked to watch their cell phones for $45 \mathrm{~min}$ to induce fatigue. The duration is designed $45 \mathrm{~min}$ because most individuals have got accustomed to this duration since childhood in school. It is confirmed that ACC, MTF, HOA4 and HOA12 present variations (Figure 3a 3d).

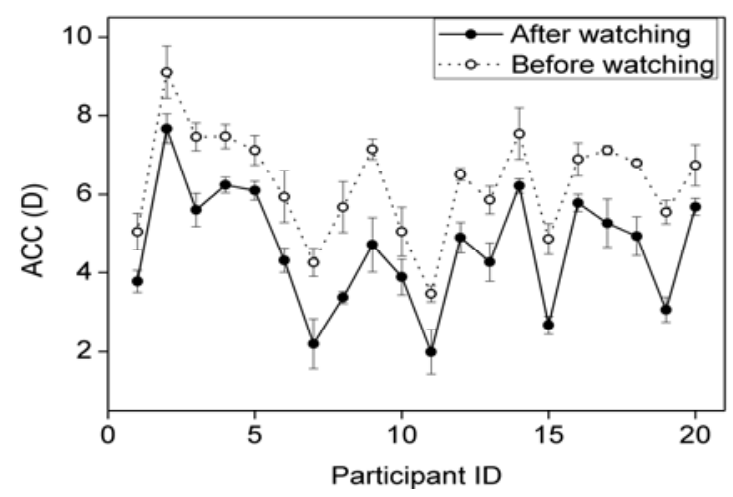

A

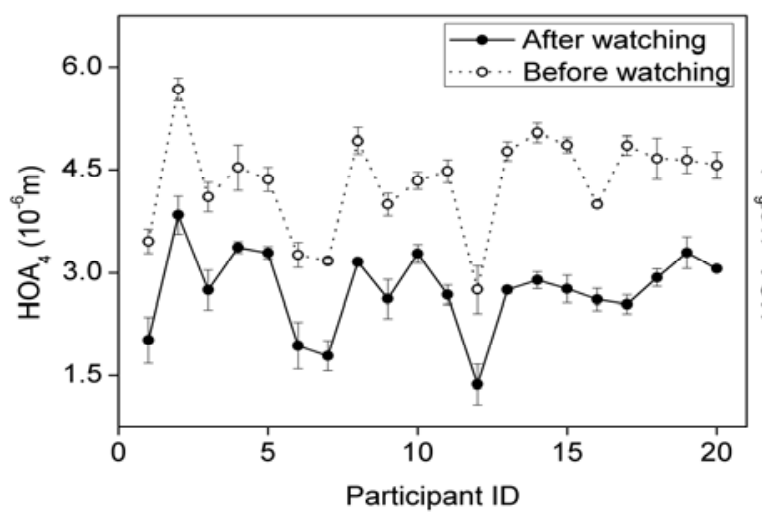

C

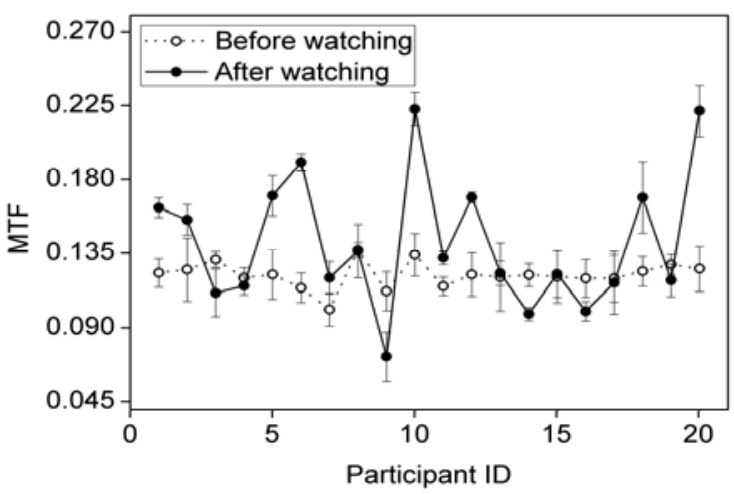

B

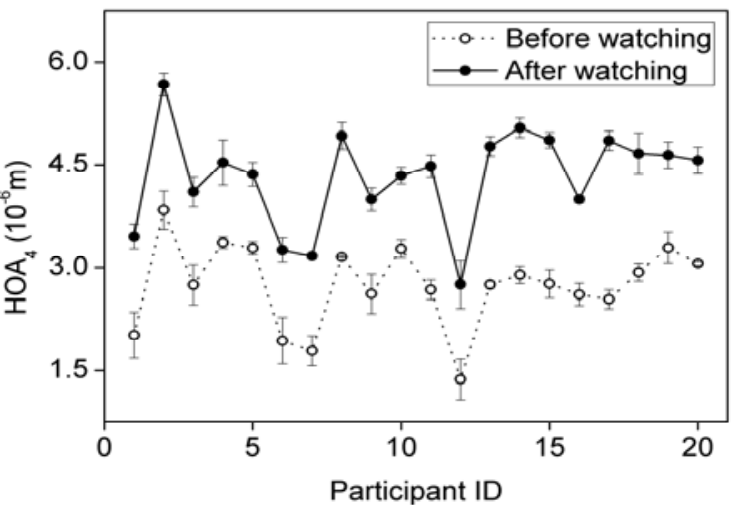

D

Figure 3 - Stability analyses on variation of (a) ACC, (b) MTF, (c) the 4th term aberration and (d) the 12th term aberration. The "before watching" dots represent the average value of parameters after 20 min rest in three days. The "after watching" dots represent the average value of parameters after $\mathbf{4 5}$ min cellphone watching in three days. Error bar represents the standard deviation of the three days measurements.

Ocular status could be described by ACC, HOAs and MTF, and the focal distance of the optical system could be described by the structural parameter AL (Mahroo, et al., 2015). These four physiological parameters are used in this study to design the evaluation formula of light-induced visual fatigue.

\subsection{Experiment process}

Process of the measurement is shown in Figure 4. First, all participants close eyes for 20 min to relax. Second, measurements were performed on participants. Third, participants executed the visual task for $45 \mathrm{~min}$. Visual task was divided into two types: lighting type and displaying type. For lighting type, experimental environment was constructed according to the standard ISO 8995, and the visual task content was Landolt rings counting. For displaying type, experimental environment was constructed according to the standard ITU-R BT.2021, and the visual task content was video watching. Last, physiological measurements were performed again. We used questionnaires for comparison. After measurements, participants were required to fill in questionnaires (Figure $4 a$ and $4 b$ ). 


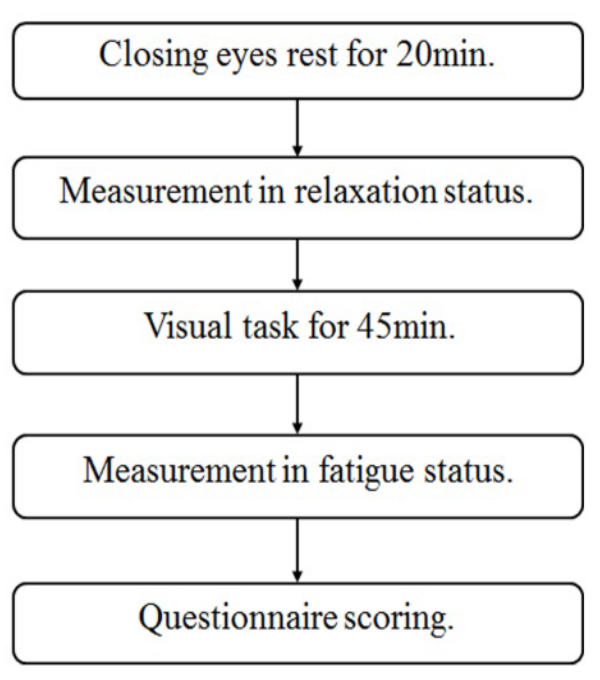

A
Subjective Score

Please score for your perception after the visual task.

$0 \sim 1$. No fatigue.

$1 \sim 2$. Slight fatigue.

2 3. Obvious fatigue within tolerance.

$3 \sim 4$. Obvious fatigue with complications.

$4 \sim 5$. Severe fatigue.

Figure 4 - Detailed information of (A) experimental process and (B) questionnaire.

\section{Results and discussion}

\subsection{Computational simulation}

Computational simulation was performed for formula construction (Burd et al., 2002). In this paper, we used Helmholtz theory for ciliary accommodation. The three types of zonulas were proposed to be connected to ciliary muscle in one reference point, and stretching displacement of the reference point was set different values to cause various deformations. We can obtained various curvature radiuses of anterior and posterior surfaces.

The simulation consists of two sections: finite element simulation of crystalline lens and optical system simulation of human eye. Finite element simulation of crystalline lens was performed by the software Abaqus 6.11 on crystalline lens. Characters were set according to the 29-year-old crystalline lens model by Burd. The crystalline lens consists of three parts: nucleus, cortex and capsule. Total number of meshes is 33,165 . Lens was proposed to be pulled by three types of zonulas with different elasticity modulus: $0.3 \mathrm{~N} / \mathrm{mm}$ for the anterior part, $0.05 \mathrm{~N} / \mathrm{mm}$ for the equator part, and $0.15 \mathrm{~N} / \mathrm{mm}$ for the posterior part. Zonula model was constructed as three springs that were pulled in one reference point. Deformation was reflected by curvature radiuses variations of anterior and posterior surfaces. The stretching displacement was in the range of $0 \sim 0.6 \mathrm{~mm}$ with $0.1 \mathrm{~mm}$ interval. For each displacement, there was a corresponding stretching force and a lens shape (Ke et al., 2017).

Optical system simulation was performed by the software Zemax 2013. Structure parameters were shown in Table 2 according to Liou. Curvature radius was defined to be negative when the curvature centre located in the right of refracting surface. We collected data of wavefront aberrations described by Zernike polynomial. Aberration variations in this study depended mainly on lens shape. Entrance pupil diameter was set $10 \mathrm{~mm}$ in uniform form. Visual field angle in $X$ and $Y$ directions were set 0 with 1 weight. Wavelength was set 0.55 um. 
Table 2 - Structure parameters of human eye model by Liou eye model (Liou et al., 1997).

\begin{tabular}{ccccc}
\hline $\begin{array}{c}\text { Refracting } \\
\text { Surface }\end{array}$ & $\begin{array}{c}\text { Curvature } \\
\text { Radius } \\
(\mathbf{m m})\end{array}$ & $\begin{array}{c}\text { Asphericity } \\
\text { Parameter }\end{array}$ & $\begin{array}{c}\text { Thickness } \\
\mathbf{( m \mathbf { m } )}\end{array}$ & $\begin{array}{c}\text { Refractive } \\
\text { Index }\end{array}$ \\
\hline $\mathbf{A}^{*}$ of Cornea & 7.77 & -0.18 & 0.50 & 1.367 \\
$\mathbf{P}^{*}$ of Cornea & 6.40 & -0.6 & 3.16 & 1.336 \\
$\mathbf{A}^{*}$ of Lens & 12.4 & -0.94 & 1.59 & Grade A \\
Gradient Surface & Infinity & -- & 2.43 & Grade P \\
$\mathbf{P}^{*}$ of Lens & -8.10 & 0.96 & 16.27 & 1.336 \\
\hline
\end{tabular}

${ }^{*} \mathrm{~A}=$ Anterior Surface, $\mathrm{P}=$ Posterior Surface.

By simulation with software Abaqus 6.11, we obtained the relation between zonular fibre stretching force (ciliary muscle force) and crystalline lens shape (curvature radius of anterior and posterior surface). Based on curvature radius of anterior and posterior surface, we can get the corresponding values of MTF and HOAs by the software Zemax 2013. We can also get the corresponding ACC by calculation. ACC represents the adjusting ability of ciliary muscle with the dimension of $D(m-1)$. By measurement, we 1can obtain three types of ACC values: ACCmax, ACCmin and ACC variation. ACCmax describes the optical power when ciliary muscle reaches the maximum tension. ACCmin describes the optical power without additional tension. ACC variation is the difference between ACCmax and ACCmin. In this study, we were sought to investigate visual fatigue formula. We focused on physiological variations ( $\triangle \mathrm{ACC}$ min, $\triangle \mathrm{MTF}$, and $\triangle \mathrm{HOAs}$ ) during the 45 min duration.
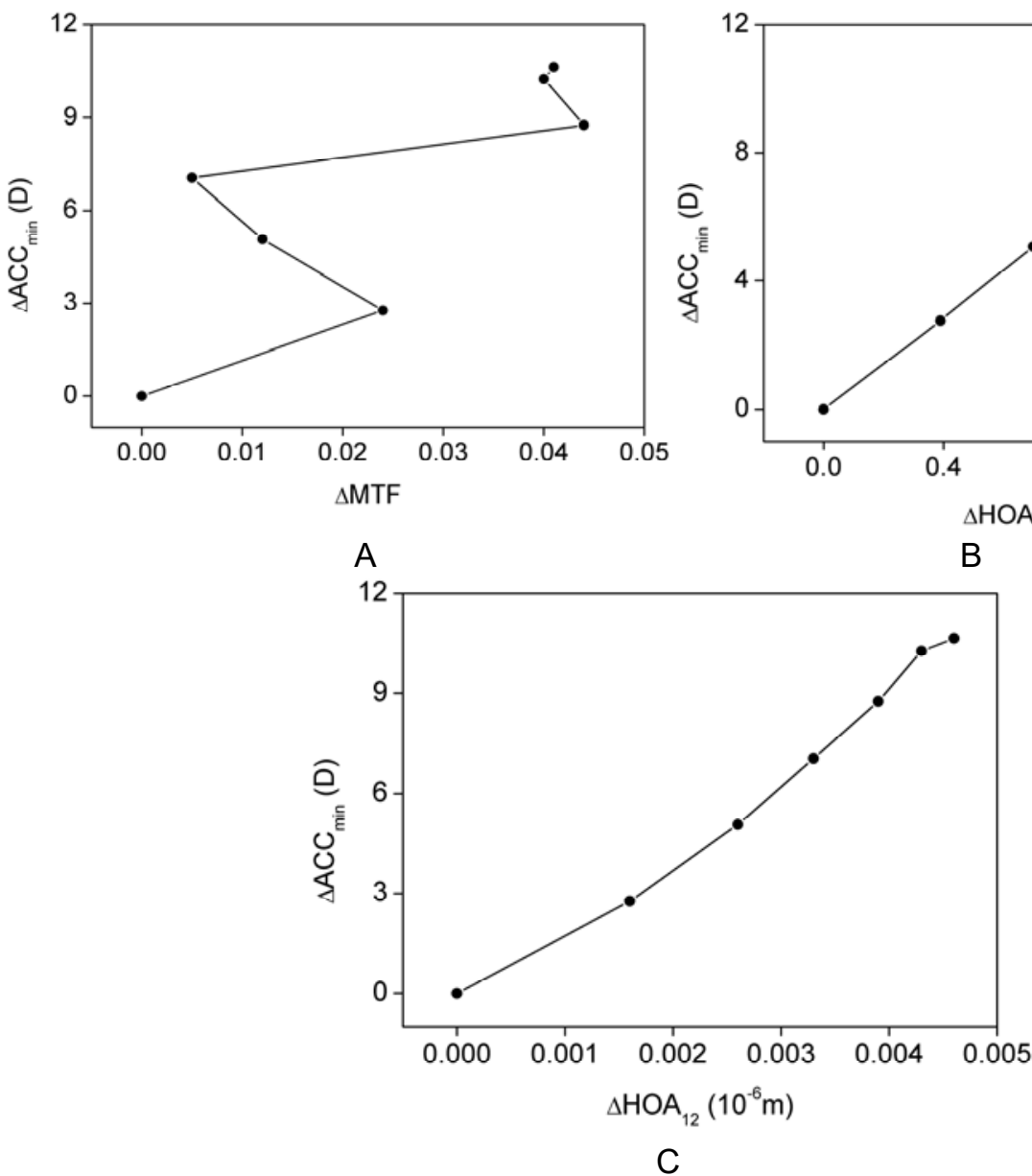

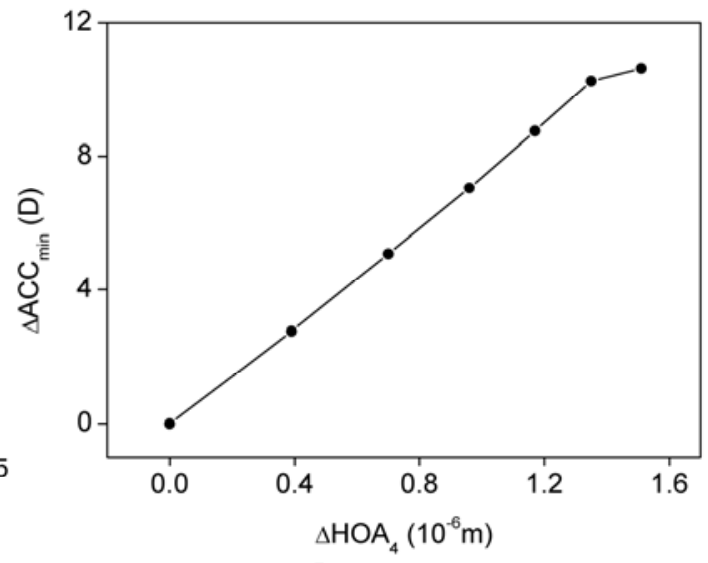

\section{with $\triangle A C C m i n$.}

Figure 5 - Variation relation of (A) MTF with $\triangle A C C$ min, (B) HOA4 with $\triangle A C C m i n,(C) \triangle H O A 12$

Variations of $\triangle M T F$ with $\triangle A C C$ min present fluctuations without regularity (Figure $5 a$ ). Variations of $\triangle \mathrm{HOA} 4$ and $\triangle \mathrm{HOA} 12$ with $\triangle \mathrm{ACC}$ in present monotonic (Figure $5 \mathrm{~b}$ and $5 \mathrm{c}$ ). During the process of accommodation, ciliary muscle stretched the crystalline lens thorough 
zonula fibres from equilibrium position (Shirachi et al., 1978). With higher visual fatigue, ciliary adjusting ability was likely to decrease during the accommodation process. In this study, we chose $\triangle \mathrm{ACC}$ min to represent visual fatigue degree. $\triangle \mathrm{ACC}$ min could be measured directly by experiment, but sole parameter was not reliable to provide accurate result. For more accurate and reliable result, $\triangle \mathrm{HOA} 4, \triangle \mathrm{HOA} 12$ and $\mathrm{AL}$ were employed together with $\triangle \mathrm{ACCmin}$ to construct the evaluation equation.

\subsection{Visual fatigue degree}

In the current study, we use $\triangle \mathrm{ACC}$ min to represent the visual fatigue degree accumulated during the 45 min duration. It is expected to figure out the expression of $\Delta \mathrm{F}$ as the function of physiological parameters that can be measured by human factor experiment. Regression analysis is performed by the simulation data. For $\triangle \mathrm{ACC}$ min as the function with independent variables of $\triangle \mathrm{HOA} 4$ and $\triangle \mathrm{HOA} 12$, multi-variable regression results are obtained $(R 2=0.996$, $\mathrm{f}=561.91, p=0)$. It is implied that linear regression is both accurate and convenient for calculation. $\triangle \mathrm{HOA} 4$ is linear functions of $\mathrm{AL}$ according to simulation. To decrease calculation errors, we employ the geometric average value. Consequently, the function of $\triangle A C C$ min could be written as

$$
\Delta \mathrm{VF}=\Delta \mathrm{ACC}_{\mathrm{min}}^{\prime}=\sqrt{\Delta \mathrm{ACC}_{\mathrm{min}} \cdot\left(b 0+k 1 \cdot \Delta \mathrm{HOA}_{4}+k 2 \cdot \Delta \mathrm{HOA}_{12}+k 3 \cdot \mathrm{AL}\right)}
$$

In the equation, $\triangle A C C$ min represents visual fatigue degree $(\triangle \mathrm{VF}), \mathrm{b} 0, \mathrm{k} 1, \mathrm{k} 2$ and $\mathrm{k} 3$ are fitting coefficients. According to simulation data, the coefficients could be fitted. However, the simulation is based on the 29-year-old human eye model, which tends to be limited and inaccurate for participants of various age ranges. For more reliable equation of visual fatigue evaluation, regression should be performed based on human factor experiment. In this study, human factor experiment was performed according to the experimental process designed in Section 2.2. Experiment information and fitting results are shown in Table 3 and Figure 6.

Table 3 - Detailed information of experiment and fitting result.

\begin{tabular}{|c|c|}
\hline Item & Information \\
\hline Participant total number & 400 \\
\hline Age distribution & $25 \sim 40$ (average 29) \\
\hline Diopter distribution & $40 \%: 0 \sim-1.00 \mathrm{D} ; 40 \%:-1.00 \mathrm{D} \sim-2.00 \mathrm{D} ; 20 \%:-2.00 \mathrm{D} \sim-5.00 \mathrm{D}$ \\
\hline $\begin{array}{l}\text { Visual acuity } \\
\text { distribution }\end{array}$ & $40 \%: 0.8 ; 60 \%: 1.0$ \\
\hline $\begin{array}{l}\text { Anisometropia above } \\
2.5 \mathrm{D}\end{array}$ & $<5 \%$ \\
\hline $\begin{array}{l}\text { Intraocular pressure } \\
\text { range }\end{array}$ & $14 \sim 20$ \\
\hline Sex & $50 \%$ : Male; 50 \%: Female \\
\hline Content & $\begin{array}{l}220 \text { participants for } 45 \text { min Landolt rings counting in LED lighting } \\
\text { pattern }(550 \mathrm{Ix}, 6500 \mathrm{~K}) \\
180 \text { participants for } 45 \mathrm{~min} \text { movie watching in LED screen } \\
\text { displaying pattern }(500 \mathrm{nits}, 6500 \mathrm{~K})\end{array}$ \\
\hline Fitting result & $\begin{array}{l}R 2=0.841, F=695.684, p=0 \\
b 0=-242, k 1=1.319, k 2=0.113, k 3=0.007\end{array}$ \\
\hline
\end{tabular}




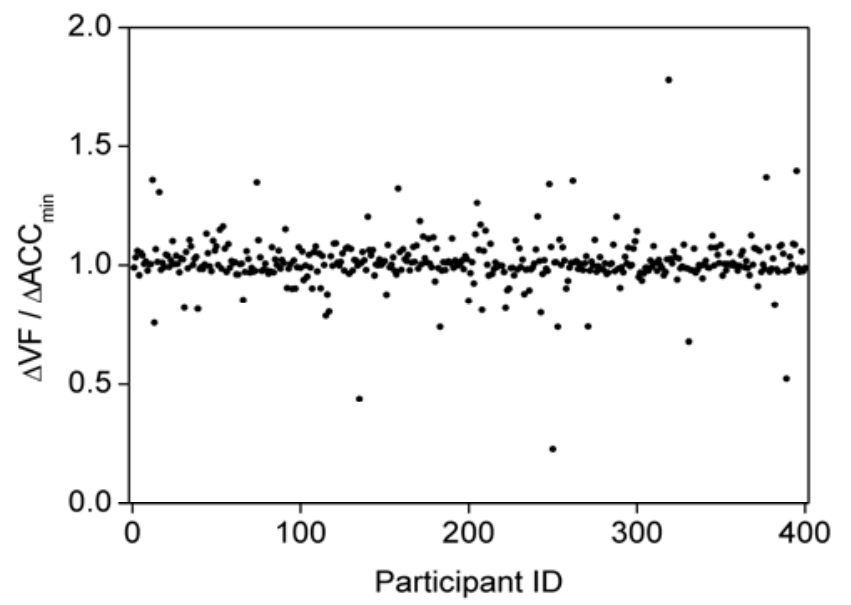

Figure 6 - Fitting result based on the human factor experiment with 400 sample capacity.

\subsection{Practical tests}

In previous discussions above, we have constructed the equation for visual fatigue evaluation during the 45 min duration based on objective physiological parameters (AL, HOA4 and HOA12) by computational simulation. To test its application, we perform human factor experiments. There are 2 experiments used in this study. In experiment 1, participants watch videos in $2 \mathrm{D}$ and $3 \mathrm{D}$ patterns respectively for $45 \mathrm{~min}$. It is known that in $3 \mathrm{D}$ pattern, visual fatigue is more likely to be accumulated since binocular vision requires imaging handling and imaging fusion in vision cortex. Visual fatigue accumulation in $3 \mathrm{D}$ pattern should be significantly higher than in 2D pattern. In experiment 2, participants count Landolt rings in classroom reading pattern with illuminances of $250 \mathrm{Ix}$ and $500 \mathrm{Ix}$ respectively.

Detailed conditions are shown in Table 4 . In experiment 2, the visual task is to read Landolt rings in a paper and count those with upward blanks.

Table 4 - Detailed information of measurements.

\begin{tabular}{ccc}
\hline Experiment & 1 & 2 \\
\hline Number & 44 & 76 \\
Sex & $50 \%$ Male, $50 \%$ Female & $50 \%$ Male, $50 \%$ Female \\
Age & $25 \sim 35($ average 29) & $25 \sim 35$ (average 26) \\
& $40 \%: 0 \sim-1.00 \mathrm{D}$ & $40 \%: 0 \sim-1.00 \mathrm{D}$ \\
Diopter & $40 \%:-1.00 \mathrm{D} \sim-2.00 \mathrm{D}$ & $40 \%:-1.00 \mathrm{D} \sim-2.00 \mathrm{D}$ \\
& $20 \%:-2.00 \mathrm{D} \sim-5.00 \mathrm{D}$ & $20 \%:-2.00 \mathrm{D} \sim-5.00 \mathrm{D}$ \\
& Pattern: screen watching $(2 \mathrm{D}$ VS 3D) & Pattern: classroom reading \\
& Luminance: 300 nits & Desk illuminance: 250 Ix VS $500 \mathrm{Ix}$ \\
Cighting & CCT: $6500 \mathrm{~K}$ & CCT: $5000 \mathrm{~K}$ \\
& Screen: $1920 * 1200$ dpi & Flicker: $<0.5 \%$ \\
& Video: "Moana" (USA, 2016) & Task: Landolt rings counting \\
& Format: mp4 & Distance: $0.4 \mathrm{~m}$ \\
& Distance: 2.5 times diagonal line & Desk: white frosted surface \\
& Standard reference: ITU-R BT.2021 & Standard reference: ISO 8995-1: 2002 \\
\hline
\end{tabular}

According to previous standards about indoor lighting, the illuminance $500 \mathrm{~lx}$ should be more proper for classroom reading. Visual fatigue accumulation in 250 Ix should be significantly higher than in $500 \mathrm{~lx}$. It is expected that our evaluation equation would provide results that are in accordance with the facts above. In addition to human factor experiments, we also collect subjective scores by questionnaire as the supplement.

For experiment 1 , visual fatigue accumulated during the 45 min duration in $3 \mathrm{D}$ pattern is significantly higher than in $2 \mathrm{D}$ pattern according to Figure $7 \mathrm{a} \sim 7 \mathrm{~b}$. Results calculated by our equation present in accordance with subjective scores as well as the fact. For experiment 3 and 4 , objective $\triangle \mathrm{VF}$ and subjective scores present opposite results. According to Figure $7 \mathrm{c}$, visual fatigue accumulated during the $45 \mathrm{~min}$ duration in $250 \mathrm{~lx}$ is significantly higher than in $500 \mathrm{Ix}$. According to Figure $7 \mathrm{~d}$, visual fatigue accumulated during the $45 \mathrm{~min}$ duration in the 
two illuminances is quite irregular. Fluctuation is quite obvious in subjective scores especially for experiments in different illuminances. But objective $\Delta V F$ is stable in both experiments. In addition, results of objective $\triangle \mathrm{VF}$ present in accordance with the fact and previous standards. Correlation analysis is performed on all data between objective $\Delta \mathrm{VF}$ and subjective scores, and correlation of 0.387 is found with significance $(p=0.003)$. Significant correlation indicates that subjective perception of visual fatigue may reflect the perception more or less, but it is likely to be affected by emotion and cognition, leading to the obvious fluctuation.

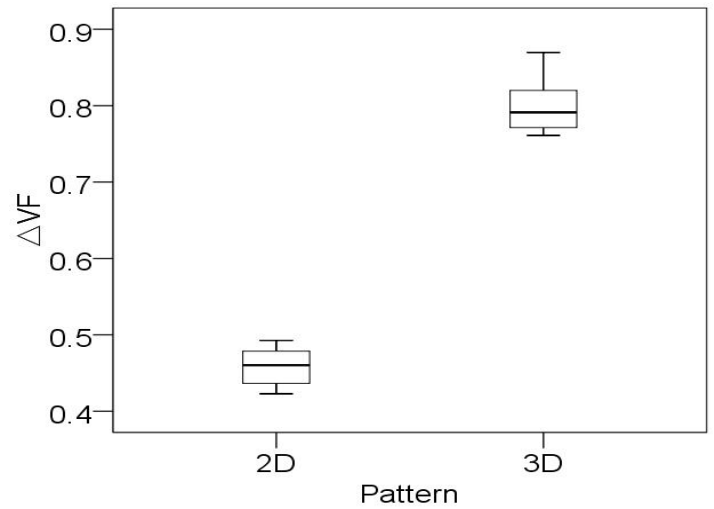

A

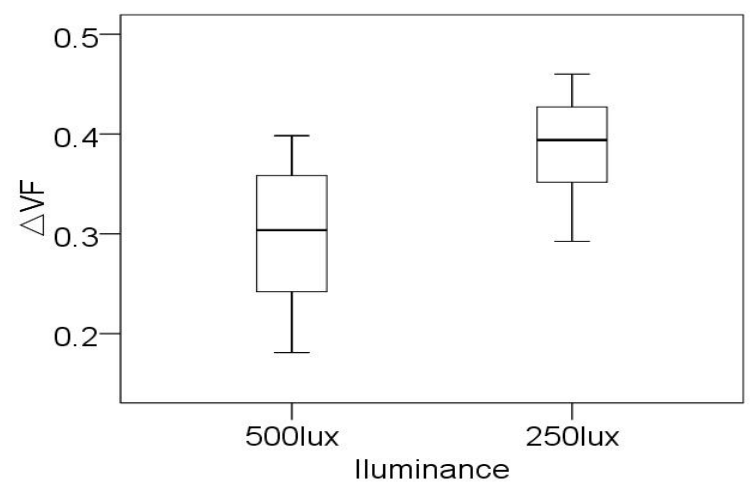

C

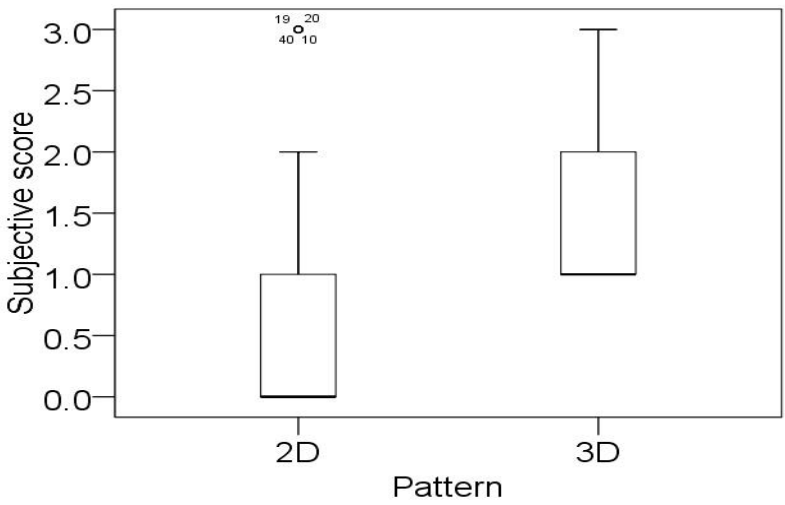

B

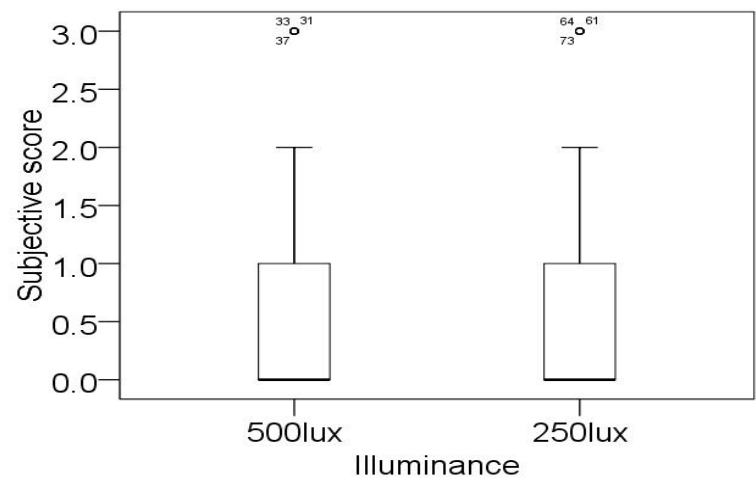

D

Figure 7 - Comparison between objective $\triangle V F$ and subjective scores. (A) $\triangle V F$ in 2D and 3D patterns. (B) Subjective scores in 2D and 3D patterns. (C) $\Delta V F$ in 250 Ix and 500 Ix. (D) Subjective scores in $\mathbf{2 5 0} \mathrm{Ix}$ and $\mathbf{5 0 0} \mathrm{Ix}$.

In this study, we develop an objective method with specified formula for light-induced visual fatigue based on ocular physiological parameters. For human eye, ocular physiological parameters are much more stable than subjective perceptions, since they are investigated by stability test prior to experiments. In addition, our results are based on large sample capacity participants. It is believed that the formula developed in the current study is relatively stable and accurate for light-induced visual fatigue evaluation.

\section{Conclusion}

In this study, we combine computational simulation and human factor experiment with large sample capacity to investigate the mechanism of light-induced visual fatigue. By analysis of structural parameters and physiological functional parameters, it is implied that light-induced visual fatigue is a special micro-variation of physiological function instead of ocular structure. According to the results of stability analysis, we found that HOAs, MTF and ACC could effectively reflect the variation regularity of assemblies in the front of human eye. Based on the duration and results of parameter selection and stability analysis, we designed a measurement method that could objectively quantify light (lighting and displaying) induced visual fatigue, resolving issues of traditional subjective questionnaire scoring such as instability and non-traceability. In addition, we constructed a calculation equation for the 
quantitative evaluation of light-induced visual fatigue. In measurements of ocular structural parameters, there are 400 participants. In stability experiment of functional parameters, there are 20 participants for the stability measurement and 20 participants for the variation measurement. In equation construction experiment, there are 400 participants. For practical test, measurements are performed on 120 participants for displaying experiment and 120 participants for lighting experiment. In total, there are 1080 measurements performed on 540 participants in this study. This method could quantify the influence of various light parameters (SPD, CCT, intensity and distribution) on visual fatigue of human eye, thus constructing the relation between physical parameters of light and physiological parameters of human eye. It is promising to provide an effective solution for quality improvement and design optimization of lighting or displaying products. More experiments on photo-biology are needed in the future to improve the efficiency and accuracy of this method. It is expected to investigate the mechanism of light-induced myopia by in the future, and figure out the method to depress myopia by optimizing light quality.

\section{References}

Abulafia A., et al. "Intraocular lens power calculation for eyes with an axial length greater than $26.0 \mathrm{~mm}$ : comparison of formulas and methods," J. Cataract Refr. Surg. 41, 548-556 (2015). (DOI: 0.1016/j.jcrs.2014.06.033)

Burd H.J., et al., "Numerical modelling of the accommodating lens," Vis. Res. 42, 2235-2251 (2002). (DOI: 10.1016/S0042-6989(02)00094-9)

Charman W.N. et al., "Wavefront Aberration of the Eye: a review," Optometry Vision Sci. 68, 574-583 (1991). (DOI: 10.1097/00006324-199108000-00002)

Ke B., et al., "The Relationship Between High-Order Aberration and Anterior Ocular Biometry During Accommodation in Young Healthy Adults," Invest. Ophth. Vis. Sci. 58, 5628 (2017). (DOI: 10.1167/iovs.17-21712)

Liou H.L., et al. "Anatomically accurate, finite model eye for optical modeling," J. Opt. Soc. Am. A. 14, 1684-1695 (1997). (DOI: 10.1364/JOSAA.14.001684)

Mahroo O.A., "Interocular asymmetries in axial length and refractive error in 4 cohorts," Ophthalmology. 122, 648-649 (2015). (DOI: 10.1016/j.ophtha.2014.10.021)

Nano TF, et al., "MTF and DQE Enhancement using an Apodized- Aperture X-Ray Detector Design," Med. Phys. 44 (2017). (DOI: 10.1002/mp.12420)

Norbäck D., et al., "Ocular symptoms and tear film break up time (BUT) among junior high school students in Penang, Malaysia - Associations with fungal DNA in school dust," Int. J. Hyg. Envir. Heal. 220, 697 (2017). (DOI: 10.1016/j.ijheh.2017.01.016)

Rubert N, et al., "SU-E-I-14: Avoiding a Common Pitfall in Performing MTF Measurements with High Resolution Kernels," Med. Phys. 42, 3244-3244 (2015). (DOI: 10.1118/1.4924011)

Shirachi D., et al., "Accommodation dynamics I. Range nonlinearity," Am. J. Optom. Phys. Opt. 55, 631 (1978). (DOI: 10.1097/00006324-197809000-00005)

Weeber H.A., et al., "Laboratory-Measured MTF of IOLs and Clinical Performance," J. Refract. Surg. 32, 211-212 (2016). (DOI: 10.3928/1081597X-20160125-01) 